\title{
Volume-staged Gamma Knife radiosurgery for orbital venous malformations
}

\author{
Dong Liu, MD, ${ }^{1}$ Yanhe Li, MD, ${ }^{1}$ Yipei Zhang, MD, ${ }^{1}$ Zhiyuan Zhang, MD, ${ }^{1}$ Guoxiang Song, MD, ${ }^{2}$ and \\ Desheng $\mathrm{Xu}, \mathrm{MD}^{1}$
}

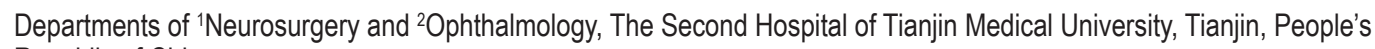
Republic of China
\end{abstract}

\begin{abstract}
OBJECTIVE This article is a preliminary evaluation of the efficacy of volume-staged Gamma Knife radiosurgery (GKRS) in the treatment of patients with orbital venous malformations (OVMs).
\end{abstract}

METHODS Twenty patients with moderate to large OVMs were treated with volume-staged GKRS between March 2005 and October 2015. The series included 8 male and 12 female patients with an average age of 22.5 years (range 9-45 years). The diagnoses were confirmed intraoperatively and at pathological examination in 14 cases and presumed in accordance with clinical and imaging findings in 6 cases. The median OVM volume was $12.2 \mathrm{~cm}^{3}\left(\right.$ range $\left.7.1-34.6 \mathrm{~cm}^{3}\right)$. The median interval between stages was 10 months (range 6-12 months). The tumor margin dose for each stage ranged from 11.0 to $13.5 \mathrm{~Gy}$. The median duration of follow-up was 45.5 months (range 18-98 months).

RESULTS Periodically scheduled MRI studies demonstrated evidence of a significant reduction of the original OVM volume in all cases. Visual acuity (VA) was preserved in 18 cases (90\%). Five patients $(25 \%)$ experienced vision improvement of varying degrees, and $13(65 \%)$ experienced long-term preservation of VA at their pre-GKRS level. Deterioration in VA was observed in only 2 cases $(10 \%)$. MRI demonstrated OVM regression after treatment in all cases, and all patients were found to have reduction of exophthalmos after volume-staged GKRS. Follow-up MRI revealed recurrence in only 1 case (5\%). Three patients (15\%) developed transient conjunctival edema.

CONCLUSIONS This retrospective investigation indicates that volume-staged GKRS provides an effective management option in selected patients with OVMs, providing excellent visual outcomes. The study adds substantial support for volume-staged GKRS as a major treatment for OVMs.

https://thejns.org/doi/abs/10.3171/2018.7.GKS18661

KEYWORDS Gamma Knife; stereotactic radiosurgery; orbit; orbital venous malformations; volume-staged radiosurgery; vascular disorders

$\mathrm{O}$ RBITAL venous malformations (OVMs) are the most common orbital vascular lesions in childhood and are characterized by impressive exophthalmos and intraorbital hemorrhage. ${ }^{5}$ The incidence of OVMs in the general population has not yet been reported, but they are considered to account for about $11 \%$ of orbital occupying diseases. The commonly used means of examination include ultrasound, CT, and MRI. Due to the complicated structure of OVMs, bleeding, calcification, and fat could be very common, and the images are usually diversified. Because OVMs often involve or wrap around the optic nerve, the difficulty of treatment is significantly increased and the lesions are prone to recurrence. Improper manage- ment may lead to a variety of serious complications, such as bleeding, injury of the optic nerve, venous thrombosis, and infection as well as increasing the risk of relapse. ${ }^{5,7}$

Volume-staged Gamma Knife radiosurgery (GKRS) has been commonly used in the management of large arteriovenous malformations, and it could potentially increase rates of obliteration and reduce damage to normal tissue in the treatment of OVMs. From March 2005 we performed volume-staged GKRS for OVMs in cases in which the lesions involved or wrapped around the optic nerve. ${ }^{9}$ The current review is devoted to the evaluation of the safety and efficacy of volume-staged GKRS as a preferred or adjuvant treatment for OVMs.

ABBREVIATIONS GKRS = Gamma Knife radiosurgery; OVM = orbital venous malformation; RION = radiation-induced optic neuropathy; VA = visual acuity.

SUBMITTED May 31, 2018. ACCEPTED July 31, 2018.

INCLUDE WHEN CITING DOI: 10.3171/2018.7.GKS18661. 


\section{Methods}

\section{Patient Characteristics}

From March 2005 to October 2015, 83 patients suffering from OVMs underwent GKRS at the Second Hospital of Tianjin Medical University. All patients were referred to us after OVMs were diagnosed by experienced ophthalmologists on the basis of typical imaging findings and clinical features and in some cases also surgical history. Twenty patients met the special selection criteria for volume-staged GKRS (i.e., moderate to large volume, lesion involving or wrapping around the optic nerve). All of the patients ( 8 male and 12 female, median age 22.5 years, range $9-45$ years) were evaluated by means of MRI and ophthalmological examinations as well as interviews or questionnaires. The diagnosis had been confirmed by pathological examination in 14 patients ( 7 of whom had undergone more than 2 prior resections), and the clinical diagnosis was presumed on the basis of clinical features and imaging findings in the other 6 patients.

All patients underwent detailed visual function and vision quality assessment before volume-staged GKRS. Visual acuity (VA) testing was performed using the standard logarithmic VA chart. Five patients had VA of 1.0 or better in the affected eyes, 8 eyes had VA of 0.6-1.0, 4 eyes had VA of $0.1-0.5$, and 3 eyes had VA of less than 0.1. All patients showed obvious proptosis in their affected eyes.

\section{Volume-Staged GKRS}

Volume-staged GKRS was performed using Leksell Gamma Knife unit model B (Elekta Instruments AB) before 2005, model C until 2014, and the Gamma Knife Perfexion from August 2014 on. Local anesthesia was induced with a mixture of lidocaine and normal saline before the Leksell $\mathrm{G}$ frame was attached, and then the patient underwent MRI or spiral CT scanning. DICOM files of MRI/CT were transferred to the Leksell GammaPlan workstation (Elekta $\mathrm{AB}$ ) for use in target delineation and dose planning. The dose plan was separated into volumetric stages demarcated by optic nerve, endeavoring to limit the maximal dose to the optic pathway to no more than 8 Gy for each stage (Fig. 1). The prescribed margin dose ranged from 11.0 to $13.5 \mathrm{~Gy}$. The median pre-GKRS OVM volume was $12.2 \mathrm{~cm}^{3}$ (range $7.1-34.6 \mathrm{~cm}^{3}$ ), and the mean volume of the first-stage GKRS was $8.8 \mathrm{~cm}^{3}$ (range $5.2-26.4 \mathrm{~cm}^{3}$ ). The second-stage GKRS was usually performed after a median interval of 10 months (range 6-12 months) with a similar prescribed margin dose and maximal dose to the visual apparatus.

\section{Follow-Up}

In all cases follow-up evaluations were performed by senior neurosurgeons or ophthalmologists who did not participate in the radiosurgical procedure. Patients were followed up at 6-month intervals for the first 2 years after GKRS and then once a year thereafter. The mean followup period was 45.5 months (range 18-98 months). The latest follow-up results were compared to the patients' baseline data for further evaluation.

\section{Statistical Analysis}

In this study, the data were analyzed and processed using SPSS version 14.0 (SPSS, Inc.).

\section{Results \\ Vision Response}

The latest data from post-GKRS VA examinations are summarized in Table 1. Five patients experienced vision improvement of varying degrees as of their latest followup. Two patients whose pre-GKRS VA was 0.2 experienced improvement after GKRS to a VA of 0.4. Three other patients whose pre-GKRS VA was $0.6-0.8$ had VA of 1.0 after GKRS. In 13 cases, the patients' VA remained stable after volume-staged GKRS (including 4 cases in which the patients' ipsilateral VA was counter finger or light perception and remained stable at their latest followup). Deterioration in VA was observed in 2 patients by $4-8$ months after their second-stage GKRS, in spite of stable VA after the first-stage GKRS. Two patients, whose preGKRS VA was 0.2 , could only count fingers via their affected eyes during the latest VA evaluation. Six patients experienced improvement in their visual field and colorcontrast function. All patients were found to have remarkable reduction of their ocular signs (such as proptosis, conjunctival hyperemia, or pain) after volume-staged GKRS.

\section{Lesion Control}

Thin-section MRI or CT scan (2.0-3.0 mm thick) was available in all 20 patients and imaging demonstrated significant reduction in lesion volume $(>75 \%)$ in all cases (100\%); this was generally observed within 6-24 months after GKRS (Fig. 1). All patients experienced OVM regression after their first-stage GKRS, and the lesion continued to shrink during the next 30 months of follow-up after the second-stage GKRS. MRI revealed conclusive evidence of OVM recurrence in only 1 patient (5\%), who subsequently underwent repeated single-stage GKRS after an interval of 48 months. Review of this patient's medical records revealed that his OVMs, involving the skin of the eyelid and inner canthus, had only received a dose of no more than $8 \mathrm{~Gy}$. No resective surgery was needed after GKRS in this group of patients.

\section{Toxicity}

Three patients experienced transient conjunctival congestion and edema for about 1 week, which responded well to steroid treatment. No patient had any signs of toxicity in their contralateral eye.

\section{Discussion}

Vascular diseases are an important part of orbital pathology. The classification of these abnormalities is difficult, and several terms are used to describe the same histological entity., ${ }^{5,6}$ The Orbital Society's hemodynamic classification divides vascular malformations into 3 categories on the basis of flow characteristics: no flow (type 1), venous flow (type 2), and arterial flow (type 3). ${ }^{5}$ Type 2 vascular malformations consist of venous and mixed venous/lymphatic channels that communicate with the ve- 

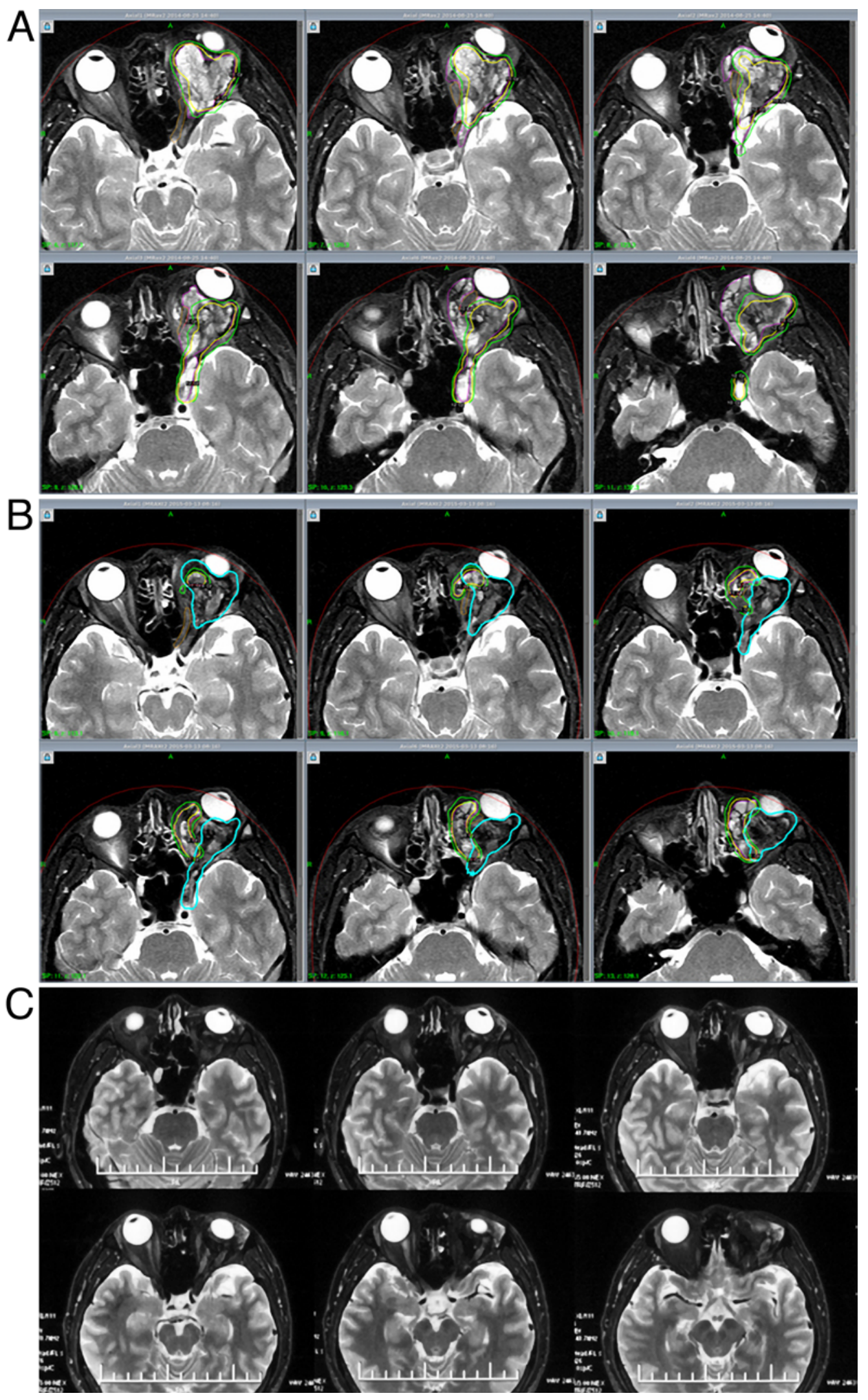

FIG. 1. Volume-staged GKRS for OVMs in a 22-year-old man, recurrence after surgical resection 5 years ago. The tumor margin dose was 12.0 Gy in the first stage and 11 Gy in the second stage. Note the regression in volume 2 years after GKRS. A: Dose plan for first-stage GKRS. B: Dose plan for second-stage GKRS. C: Axial $T_{2}$-weighted MR images obtained 2 years after secondstage GKRS. 
TABLE 1. Aggregate clinical outcome and lesion control in 20 patients with OVMs

\begin{tabular}{lc}
\hline \multicolumn{1}{c}{ Variable } & Value \\
\hline Follow-up period (mos) & \\
\hline Median & 45.5 \\
\hline Range & $18-98$ \\
\hline Visual outcome & $5(25 \%)$ \\
\hline Improved & $13(65 \%)$ \\
\hline Stable & $2(10 \%)$ \\
\hline Worse & $20(100 \%)$ \\
\hline Lesion control & $0(0 \%)$ \\
\hline Regression (reduction in lesion size) & $1(5 \%)$ \\
\hline Stability or progression & $3(15 \%)$ \\
\hline Treatment-related toxicity
\end{tabular}

Data are presented as number of patients (\%) unless otherwise indicated.

nous system. The Orbital Society divides type 2 venous flow malformations into 3 subclasses: nondistensible venous, distensible venous, and distensible combined venous and lymphatic malformations. The behavior of nondistensible venous malformations is clinically similar to that of type 1 lymphatic malformations, with frequent episodes of spontaneous thrombosis and hemorrhage. Doppler and contrast imaging show evidence of venous flow. Management of lesions with significant venous communication requires significantly greater caution because of the higher risk of venous thrombosis and bleeding. Improper treatment may lead to a variety of serious complications and risk of relapse. Although the identification and management of OVMs is generally performed by an ophthalmologist, many OVM processes, for instance, lesions within the orbital apex or those enveloping the optic apparatus in patients with useful VA, require the involvement of other specialties. Despite rapid advances in ophthalmic surgery, surgery-related complications associated with nerve injury still haunt the majority of orbital surgeons. The deterioration of symptoms after lesion excision is very common; therefore, more and more patients are now likely to prefer the least risky option. ${ }^{1,8}$ A minimally invasive and effective therapy is needed for such patients with OVMs.

\section{Dose Selection}

A number of factors need to be considered for the choice of radiosurgical prescription dose, including pathological properties, lesion volume, location relative to the optic apparatus, the patient's visual function, and radiosensitivity. ${ }^{2}$ Since histopathology of venous malformations reveals abnormal thin-walled veins of varying sizes, with stromal tissue distributed between vessels, ${ }^{5}$ a $12-18$ Gy prescription dose seems to be sufficient. We believe, based on our experience, that volume-staged GKRS represents a remarkable advance in OVM management. In the present series, the median prescription peripheral dose ranged from 11 to $13.5 \mathrm{~Gy}$. All patients experienced OVM shrinkage after the first-stage GKRS, and the lesion con- tinued to shrink during the next 30 months of follow-up after the second-stage GKRS.

\section{Optic Apparatus Tolerance}

In view of the fact that OVMs often involve or wrap around the optic nerve, the difficulty of treatment is significantly increased and the lesions are prone to recurrence. For patients with good vision function in the affected eye, one must consider how to avoid radiation-induced optic neuropathy (RION) as well as how to control the OVM. Studies on optic nerve radiation damage have shown that a single dose of 8-10 Gy is safe, and Leber et al. ${ }^{3}$ retrospectively studied the relationship between the dose tolerance of the visual pathway in front of the lateral geniculate body and RION in 50 patients with benign tumors. They found that the incidence of RION was $26.7 \%$ when the dose to the optic nerve was 10-15 Gy, and the incidence increased to $77.8 \%$ when the dose was $\geq 15 \mathrm{~Gy}$. Mayo et al. ${ }^{4}$ also indicated that the risk of RION appeared to increase with a dose of more than $60 \mathrm{~Gy}$ at $1.8 \mathrm{~Gy} /$ fraction or more than $12 \mathrm{~Gy}$ for a single fraction. Xu et al. ${ }^{9}$ speculated that the optic nerve within the orbit may be able to endure a higher radiation dose than the cranial part. In our series, only 2 patients suffered from deterioration in VA by $4-8$ months after the second-stage GKRS. The pre-GKRS VA for these 2 patients was 0.2 , and the OVM volume was 22.7 $\mathrm{cm}^{3}$ in one case and $31.5 \mathrm{~cm}^{3}$ in the other. We speculate that the higher radiation tolerance of the intraorbital optic nerve may be related to the attenuation of irradiation dose by surrounding soft tissues and bones.

\section{Conclusions}

This retrospective investigation indicates that volumestaged GKRS provides an effective management option for OVMs in selected patients, achieving and maintaining excellent visual function. The study adds substantial evidence for the use of volume-staged GKRS as a major treatment for OVM.

\section{Acknowledgments}

We thank Li-Gao Zheng, Yi-Guang Lin, Guo-Kai Wang, and Wei-Zheng Guo for their generous assistance and support of this research.

\section{References}

1. Gündüz K, Karcioglu ZA: Vascular tumors, in Karcioglu ZA (ed): Orbital Tumors. New York: Springer, 2015, pp 155-181

2. Kirkpatrick JP, van der Kogel AJ, Schultheiss TE: Radiation dose-volume effects in the spinal cord. Int J Radiat Oncol Biol Phys 76 (3 Suppl):S42-S49, 2010

3. Leber KA, Berglöff J, Pendl G: Dose-response tolerance of the visual pathways and cranial nerves of the cavernous sinus to stereotactic radiosurgery. J Neurosurg 88:43-50, 1998

4. Mayo C, Martel MK, Marks LB, Flickinger J, Nam J, Kirkpatrick J: Radiation dose-volume effects of optic nerves and chiasm. Int J Radiat Oncol Biol Phys 76 (3 Suppl):S28S35, 2010

5. Naggara O, Koskas P, Lafitte F, Heran F, Piekarski JD, Meder JF, et al: [Vascular tumours and malformation of the orbit.] J Radiol 87:17-27, 2006 (Fr) 
6. Rootman J, Heran MKS, Graeb DA: Vascular malformations of the orbit: classification and the role of imaging in diagnosis and treatment strategies. Ophthal Plast Reconstr Surg 30:91-104, 2014

7. Sullivan TJ, Wright JE: Non-traumatic orbital haemorrhage. Clin Experiment Ophthalmol 28:26-31, 2000

8. Taban M, Perry JD: Classification of orbital tumors, in Singh AD, Damato BE, Pe'er J, et al (eds): Essentials of Ophthalmic Oncology. Thorofare, NJ: Slack, 2009, p 214

9. Xu D, Liu D, Zhang Z, Zhang Y, Li Y, Liu X, et al: Gamma Knife surgery in the management of orbital tumors. J Neurosurg 113 Suppl:34-38, 2010

\section{Disclosures}

The authors report no conflict of interest concerning the materials or methods used in this study or the findings specified in this paper.

\section{Author Contributions}

Conception and design: Liu, Song, Xu. Acquisition of data: Liu, Li, Y Zhang, Z Zhang, Song. Analysis and interpretation of data: Liu, Li, Z Zhang. Drafting the article: Liu. Critically revising the article: Liu. Reviewed submitted version of manuscript: Liu, $\mathrm{Xu}$. Approved the final version of the manuscript on behalf of all authors: Liu. Statistical analysis: Liu. Administrative/technical/ material support: Liu, Z Zhang. Study supervision: Liu.

\section{Supplemental Information}

Previous Presentations

Portions of the work were previously presented in abstract form at the 19th Leksell Gamma Knife Society Meeting, Dubai, United Arab Emirates, March 4-8, 2018.

\section{Correspondence}

Dong Liu: Gamma Knife Center, The Second Hospital of Tianjin Medical University, Tianjin, China. liudong@tmu.edu.cn. 